\title{
Occasionally Weakly Compatible Mappings in Quasi-Gauge Space
}

\author{
Arihant Jain \\ Shri Guru Sandipani Girls' Institute of \\ Professional Studies, \\ Ujjain (M.P.), India
}

\author{
Basant Chaudhary \\ Research Scholar, \\ Department of Applied Mathematics, \\ Mewar University, Chittorgarh (Raj.), India
}

\begin{abstract}
The present paper deals with the common fixed point theorem for occasionally weak compatible mappings in Quasi-gauge space. Moreover, we improve and generalize the results of Rao et. al. [8, 9]. An illustrative example is also given.
\end{abstract}

Keywords: Common fixed point, weakly compatible mappings, Quasi-gauge space.

\section{INTRODUCTION}

In the year 1973, Reilly [10] was the first to introduce the concept of Quasi-gauge space. Afterwards, Antony et. al. [3] gave a generalization of a common fixed point theorem of Fisher [5] for Quasigauge spaces. Pathak et. al. [7] proved fixed point theorems for compatible mappings of type (P). Rao and Murthy [8] extended results on common fixed points of self maps by replacing the domain "complete metric space" with "Quasi-gauge space". But in both theorems continuity of any mapping was the necessary condition for the existence of the fixed point. Afterwards Rao et. al. [9] extended the results of Rao and Murthy [8] under weaker conditions. Jain et. al. [4] improve the results of Rao et.al. [8] and showed that the continuity of any mapping for the existence of the fixed point is not required. In this paper, we introduce the concept of occasionally weakly mappings in Quasi-Gauge space after motivated by the result of Al-Thagafi and Shahzad [1].

\section{Preliminaries}

Definition 1.1. [10] A Quasi-pseudo-metric on a set $X$ is a non-negative real valued function $p$ on $\mathrm{X} \times \mathrm{X}$ such that

(i) $\mathrm{p}(\mathrm{x}, \mathrm{x})=0$ for all $\mathrm{x} \in \mathrm{X}$.

(ii) $\mathrm{p}(\mathrm{x}, \mathrm{z}) \leq \mathrm{p}(\mathrm{x}, \mathrm{y})+\mathrm{p}(\mathrm{y}, \mathrm{z})$ for all $\mathrm{x}, \mathrm{y}, \mathrm{z} \in \mathrm{X}$.

Definition 1.2. [10] A Quasi-gauge structure for a topological space $(X, T)$ is a family $P$ of quasipseudo-metrics on $\mathrm{X}$ such that $\mathrm{T}$ has as a sub-base the family

$$
\{\mathrm{B}(\mathrm{x}, \mathrm{p}, \varepsilon): \mathrm{x} \in \mathrm{X}, \mathrm{p} \in \mathrm{P}, \varepsilon>0\}
$$

where $\mathrm{B}(\mathrm{x}, \mathrm{p}, \varepsilon)$ is the set $\{\mathrm{y} \in \mathrm{X}: \mathrm{p}(\mathrm{x}, \mathrm{y})<\varepsilon\}$. If a topological space has a Quasi-gauge structure, it is called a Quasi-gauge space.

Definition 1.3. [10] A sequence $\left\{x_{n}\right\}$ in a Quasi-gauge space $(X, P)$ is said to be P-Cauchy, if for each $\varepsilon>0$ and $\mathrm{p} \in \mathrm{P}$ there is an integer $\mathrm{k}$ such that $\mathrm{p}\left(\mathrm{x}_{\mathrm{m}}, \mathrm{x}_{\mathrm{n}}\right)<\varepsilon$ for all $\mathrm{m}, \mathrm{n} \geq \mathrm{k}$.

Definition 1.4. [10] A Quasi-gauge space $(X, P)$ is sequentially complete if and only if every $\mathrm{P}$-Cauchy sequence in $\mathrm{X}$ is convergent in $\mathrm{X}$.

We now propose the following characterization. Let $(X, P)$ be a Quasi-gauge space. $X$ is a $T_{0}$ space if and only if $\mathrm{p}(\mathrm{x}, \mathrm{y})=\mathrm{p}(\mathrm{y}, \mathrm{x})=0$ for all $\mathrm{p}$ in P implies $\mathrm{x}=\mathrm{y}$. 
Antony [2] introduced the concept of weak compatibility for a pair of mappings on Quasigauge Space.

Definition 1.5. [2] Let $(X, P)$ be a Quasi-gauge space. The self maps $f$ and $g$ are said to be (f,g) weak compatible if $\lim _{n \rightarrow \infty} \operatorname{gfx}_{n}=f z$ for some $z \in X$ whenever $x_{n}$ is sequence in $X$ such that $\lim _{n \rightarrow \infty} f_{n}=$ $\lim _{n \rightarrow \infty}{g x_{n}}_{n}=z$ and $\lim _{n \rightarrow \infty} f_{g x} x_{n}=\lim _{n \rightarrow \infty} f f f_{n}=f z$.

$f$ and $g$ are said to be weak compatible to each other if (f,g) and $(g, f)$ are weak compatible.

Definition 1.6. [9] Let (X, P) be a Quasi-gauge space. The self maps A and S are said to be weakly compatible if and only if they commute at their coincidence points, i.e. if $\mathrm{Ap}=\mathrm{Sp}$ for some $\mathrm{p} \in \mathrm{X}$ then $\mathrm{ASp}=\mathrm{SAp}$.

This is weaker than the previous one (see example 1.7 [9]).

Motivated by Al-Thagafi and Shahzad [1], who introduced the concept of occasionally weakly compatible maps, we introduce the concept of occasionally weakly compatible maps in Qausi-gauge space as follows :

Definition 1.7. Let $(X, P)$ be a Quasi-gauge space. The self maps $A$ and $S$ are said to be occasionally weakly compatible (owc) if and only if there is a point $\mathrm{x}$ in $\mathrm{X}$ which is a coincidence point of $\mathrm{A}$ and $\mathrm{S}$ at which $\mathrm{A}$ and $\mathrm{S}$ both commute.

The following is an example of pair of self maps in a Quasi-gauge space $(\mathrm{X}, \mathrm{P})$ which are occasionally weakly compatible but not weakly compatible.

Example 1.1. Let $(X, P)$ be Quasi-gauge space, where $X=[0,4]$ with the usual metric d. Define self maps $A$ and $S$ by $A x=4 x$ and $S x=x^{2}$ for all $x \in X$ then $A x=S x$ for $x=0$ and 4. But $A S(0)=S A(0)$ and $\mathrm{AS}(4) \neq \mathrm{SA}(4)$.

Thus, A and S are occasionally weakly compatible but not weakly compatible.

From the above example it is obvious that the concept of occasionally weak compatibility is more general than that of weak compatibility.

The following is useful in establishing our result.

Lemma 1.1. [6] Suppose that $\psi:[0, \infty) \rightarrow[0, \infty)$ is non-decreasing and upper semi-continuous from the right. If $\psi(\mathrm{t})<\mathrm{t}$ for every $\mathrm{t}>0$, then $\lim \psi^{\mathrm{n}}(\mathrm{t})=0$.

Now, we establish a common fixed point theorem for four self maps which generalizes the results of Rao et. al. [8, 9].

\section{MAin ReSUlt}

Theorem 2.1. Let J, K, L and $M$ be self maps on a left (right) sequentially complete Quasi-Gauge $\mathrm{T}_{0}$ space $(\mathrm{X}, \mathrm{P})$ such that

(2.1) $(\mathrm{J}, \mathrm{L})$ and $(\mathrm{K}, \mathrm{M})$ are occasionally weakly compatible pair of mappings;

$$
\begin{aligned}
& \mathrm{M}(\mathrm{X}) \subseteq \mathrm{J}(\mathrm{X}) \text { and } \mathrm{L}(\mathrm{X}) \subseteq \mathrm{K}(\mathrm{X}) \\
& \max \left\{\mathrm{p}^{2}(\mathrm{Lx}, \mathrm{My}), \mathrm{p}^{2}(\mathrm{My}, \mathrm{Lx})\right\} \\
& \leq \phi\left\{\mathrm{p}\left(\mathrm{Jx}_{\mathrm{x}}, \mathrm{Lx}\right) \mathrm{p}(\mathrm{Ky}, \mathrm{My}), \mathrm{p}(\mathrm{Jx}, \mathrm{My}) \mathrm{p}(\mathrm{Ky}, \mathrm{Lx}), \mathrm{p}(\mathrm{Jx}, \mathrm{Lx}) \mathrm{p}\left(\mathrm{Jx}_{\mathrm{x}}, \mathrm{My}\right),\right. \\
& \mathrm{p}(\mathrm{Ky}, \mathrm{Lx}) \mathrm{p}(\mathrm{Ky}, \mathrm{My}), \mathrm{p}(\mathrm{Ky}, \mathrm{Lx}) \mathrm{p}(\mathrm{Jx}, \mathrm{Lx}), \mathrm{p}(\mathrm{Ky}, \mathrm{My}) \mathrm{p}(\mathrm{Jx}, \mathrm{My})\}
\end{aligned}
$$

for all $\mathrm{x}, \mathrm{y} \in \mathrm{X}$ and for all $\mathrm{p}$ in $\mathrm{P}$, where $\phi:[0, \infty)^{6} \rightarrow(0,+\infty)$ satisfies the following:

$\phi$ is non-decreasing and upper semi-continuous in each coordinate variable and for each $t>0$ :

$\psi(\mathrm{t})=\max \{\phi(\mathrm{t}, 0,2 \mathrm{t}, 0,0,2 \mathrm{t}), \phi(\mathrm{t}, 0,0,2 \mathrm{t}, 2 \mathrm{t}, 0), \phi(0, \mathrm{t}, 0,0,0,0)$,

$$
\phi(0,0,0,0,0, \mathrm{t}), \phi(0,0,0,0, \mathrm{t}, 0)\}<\mathrm{t} .
$$


Then $\mathrm{J}, \mathrm{K}, \mathrm{L}$ and $\mathrm{M}$ have a unique common fixed point.

Proof. Let $\mathrm{x}_{0}$ be an arbitrary point in $\mathrm{X}$. Since (2.1) holds we can choose $\mathrm{x}_{1}, \mathrm{x}_{2}$ in $\mathrm{X}$ such that $\mathrm{Kx}_{1}=$ $\mathrm{Lx}_{0}$ and $\mathrm{Jx}_{2}=\mathrm{Mx}_{1}$.

In general we can choose $\mathrm{x}_{2 \mathrm{n}+1}$ and $\mathrm{x}_{2 \mathrm{n}+2}$ in $\mathrm{X}$ such that

$$
\mathrm{y}_{2 \mathrm{n}}=\mathrm{Kx}_{2 \mathrm{n}+1}=\mathrm{Lx}_{2 \mathrm{n}} \text { and } \mathrm{y}_{2 \mathrm{n}+1}=\mathrm{Jx}_{2 \mathrm{n}+2}=\mathrm{Mx}_{2 \mathrm{n}+1} ; \mathrm{n}=0,1,2, \ldots \text {. }
$$

We denote $d_{n}=p\left(y_{n}, y_{n+1}\right)$ and $e_{n}=p\left(y_{n+1}, y_{n}\right)$; now applying (2.3) we get

$$
\begin{aligned}
& \max \left\{\mathrm{d}_{2 \mathrm{n}+2}^{2}, \mathrm{e}_{2 \mathrm{n}+2}^{2}\right\} \\
& =\max \left\{\mathrm{p}^{2}\left(\mathrm{Lx}_{2 \mathrm{n}+2}, \mathrm{Mx}_{2 \mathrm{n}+3}\right), \mathrm{p}^{2}\left(\mathrm{Mx}_{2 \mathrm{n}+3}, \mathrm{Lx}_{2 \mathrm{n}+2}\right)\right\}
\end{aligned}
$$

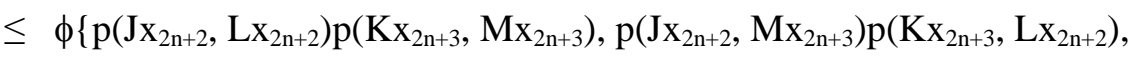

$$
\begin{aligned}
& \mathrm{p}\left(\mathrm{Jx}_{2 \mathrm{n}+1}, \mathrm{Lx}_{2 \mathrm{n}+2}\right) \mathrm{p}\left(\mathrm{Jx}_{2 \mathrm{n}+2}, \mathrm{Mx}_{2 \mathrm{n}+1}\right), \mathrm{p}\left(\mathrm{Kx}_{2 \mathrm{n}+1}, \mathrm{Lx}_{2 \mathrm{n}+2}\right) \mathrm{p}\left(\mathrm{Kx}_{2 \mathrm{n}+2}, \mathrm{Mx}_{2 \mathrm{n}+3}\right), \\
& \left.\mathrm{p}\left(\mathrm{Kx}_{2 \mathrm{n}+3}, \mathrm{Lx}_{2 \mathrm{n}+2},\right) \mathrm{p}\left(\mathrm{Jx}_{2 \mathrm{n}+2}, \mathrm{Lx}_{2 \mathrm{n}+1}\right), \mathrm{p}\left(\mathrm{Kx}_{2 \mathrm{n}+3}, \mathrm{Mx}_{2 \mathrm{n}+3}\right) \mathrm{p}\left(\mathrm{Jx}_{2 \mathrm{n}+2}, \mathrm{Mx}_{2 \mathrm{n}+3}\right)\right\} \\
& =\phi\left\{\mathrm{p}\left(\mathrm{y}_{2 \mathrm{n}+1}, \mathrm{y}_{2 \mathrm{n}+2}\right) \mathrm{p}\left(\mathrm{y}_{2 \mathrm{n}+2}, \mathrm{y}_{2 \mathrm{n}+3}\right), \mathrm{p}\left(\mathrm{y}_{2 \mathrm{n}+1}, \mathrm{y}_{2 \mathrm{n}+3}\right) \mathrm{p}\left(\mathrm{y}_{2 \mathrm{n}+2}, \mathrm{y}_{2 \mathrm{n}+2}\right)\right. \text {, } \\
& p\left(y_{2 n+1}, y_{2 n+2}\right) p\left(y_{2 n+1}, y_{2 n+3}\right), p\left(y_{2 n+2}, y_{2 n+2}\right) p\left(y_{2 n+2}, y_{2 n+3}\right), \\
& \left.\mathrm{p}\left(\mathrm{y}_{2 \mathrm{n}+2}, \mathrm{y}_{2 \mathrm{n}+2}\right) \mathrm{p}\left(\mathrm{y}_{2 \mathrm{n}+1}, \mathrm{y}_{2 \mathrm{n}+2}\right), \mathrm{p}\left(\mathrm{y}_{2 \mathrm{n}+2}, \mathrm{y}_{2 \mathrm{n}+3}\right) \mathrm{p}\left(\mathrm{y}_{2 \mathrm{n}+1}, \mathrm{y}_{2 \mathrm{n}+3}\right)\right\} \\
& \leq \phi\left\{d_{2 n+1} d_{2 n+2}, 0, d_{2 n+1}\left(d_{2 n+1}+d_{2 n+2}\right), 0,0, d_{2 n+2}\left(d_{2 n+1}+d_{2 n+2}\right)\right\} .
\end{aligned}
$$

If $\mathrm{d}_{2 \mathrm{n}+2}>\mathrm{d}_{2 \mathrm{n}+1}$ then

$$
\max \left\{\mathrm{d}^{2}{ }_{2 \mathrm{n}+2}, \mathrm{e}^{2}{ }_{2 \mathrm{n}+2}\right\} \leq \phi\left\{\mathrm{d}^{2}{ }_{2 \mathrm{n}+2}, 0,2 \mathrm{~d}^{2}{ }_{2 \mathrm{n}+2}, 0,0,2 \mathrm{~d}^{2}{ }_{2 \mathrm{n}+2}\right\}<\mathrm{d}^{2}{ }_{2 \mathrm{n}+2},
$$

by (2.4) a contradiction; hence $\mathrm{d}_{2 \mathrm{n}+2} \leq \mathrm{d}_{2 \mathrm{n}+1}$. Similarly, we get

$$
\mathrm{d}_{2 \mathrm{n}+1} \leq \mathrm{d}_{2 \mathrm{n}} .
$$

By (2.6) and (2.7)

$$
\max \left\{\mathrm{d}^{2}{ }_{2 \mathrm{n}+2}, \mathrm{e}^{2}{ }_{2 \mathrm{n}+2},\right\} \leq \phi\left\{\mathrm{d}^{2}{ }_{2 \mathrm{n}+1}, 0,2 \mathrm{~d}^{2}{ }_{2 \mathrm{n}+1}, 0,0,2 \mathrm{~d}^{2}{ }_{2 \mathrm{n}+1}\right\} .
$$

Similarly, we get

$$
\begin{aligned}
\max \left\{\mathrm{d}^{2}{ }_{2 \mathrm{n}+1}, \mathrm{e}^{2}{ }_{2 \mathrm{n}+1}\right\} & \leq \phi\left\{\mathrm{d}^{2}{ }_{2 \mathrm{n}}, 0,0,2 \mathrm{~d}^{2}{ }_{2 \mathrm{n}}, 2 \mathrm{~d}^{2}{ }_{2 \mathrm{n}}, 0\right\} . \\
\leq & \psi\left\{\mathrm{p}^{2}\left(\mathrm{y}_{2 \mathrm{n}}, \mathrm{y}_{2 \mathrm{n}+1}\right)\right\} .
\end{aligned}
$$

So

$$
\mathrm{d}^{2}{ }_{\mathrm{n}}=\mathrm{p}^{2}\left(\mathrm{y}_{\mathrm{n}}, \mathrm{y}_{\mathrm{n}+1}\right) \leq \psi\left\{\mathrm{p}^{2}\left(\mathrm{y}_{\mathrm{n}+1}, \mathrm{y}_{\mathrm{n}}\right)\right\} \leq \ldots \leq \psi^{\mathrm{n}-1}\left\{\mathrm{p}^{2}\left(\mathrm{y}_{1}, \mathrm{y}_{2}\right)\right\}
$$

and

$$
\mathrm{e}^{2}{ }_{\mathrm{n}}=\mathrm{p}^{2}\left(\mathrm{y}_{\mathrm{n}+1}, \mathrm{y}_{\mathrm{n}}\right) \leq \psi\left\{\mathrm{p}^{2}\left(\mathrm{y}_{\mathrm{n}-1}, \mathrm{y}_{\mathrm{n}}\right)\right\} \leq \ldots \leq \psi^{\mathrm{n}-1}\left\{\mathrm{p}^{2}\left(\mathrm{y}_{1}, \mathrm{y}_{2}\right)\right\} .
$$

Hence by Lemma 1.1 and from (2.10) and (2.11), we obtain

$$
\lim _{\mathrm{n} \rightarrow \infty} \mathrm{d}_{\mathrm{n}}=\lim _{\mathrm{n} \rightarrow \infty} \mathrm{e}_{\mathrm{n}}=0 .
$$

Now we prove $\left\{y_{n}\right\}$ is a P-Cauchy sequence. To show this, it is sufficient to show that $\left\{y_{2 n}\right\}$ is PCauchy. Suppose $\left\{\mathrm{y}_{2 \mathrm{n}}\right\}$ is not a P-Cauchy sequence then there exists an $\varepsilon>0$ such that for each positive integer $2 \mathrm{k}$ there exist positive integers $2 \mathrm{~m}(\mathrm{k})$ and $2 \mathrm{n}(\mathrm{k})$ such that for some $\mathrm{p}$ in $\mathrm{P}$,

$$
\mathrm{p}\left(\mathrm{y}_{2 \mathrm{n}(\mathrm{k})}, \mathrm{y}_{2 \mathrm{~m}(\mathrm{k})}\right)>\varepsilon \text { for } 2 \mathrm{~m}(\mathrm{k})>2 \mathrm{n}(\mathrm{k})>2 \mathrm{k}
$$

and

$$
\mathrm{p}\left(\mathrm{y}_{2 \mathrm{~m}(\mathrm{k})}, \mathrm{y}_{2 \mathrm{n}(\mathrm{k})}\right)>\varepsilon \text { for } 2 \mathrm{~m}(\mathrm{k})>2 \mathrm{n}(\mathrm{k})>2 \mathrm{k}
$$


for each positive even integer $2 \mathrm{k}$, let $2 \mathrm{~m}(\mathrm{k})$ be the least positive even integer exceeding $2 \mathrm{n}(\mathrm{k})$ and satisfying (2.13); hence $\mathrm{p}\left(\mathrm{y}_{2 \mathrm{n}(\mathrm{k})}, \mathrm{y}_{2 \mathrm{~m}(\mathrm{k})-2}\right) \leq \varepsilon$ then for each even integer $2 \mathrm{k}$,

$$
\begin{aligned}
\varepsilon & <\mathrm{p}\left(\mathrm{y}_{2 \mathrm{n}}(\mathrm{k}), \mathrm{y}_{2 \mathrm{~m}}(\mathrm{k})\right) \\
& \leq \mathrm{p}\left(\mathrm{y}_{2 \mathrm{n}(\mathrm{k})}, \mathrm{y}_{2 \mathrm{~m}(\mathrm{k})-2}\right)+\left(\mathrm{d}_{2 \mathrm{~m}(\mathrm{k})-2}+\mathrm{d}_{2 \mathrm{~m}(\mathrm{k})-1}\right) .
\end{aligned}
$$

From (2.12) and (2.15), we obtain $\lim _{\mathrm{n} \rightarrow \infty} \mathrm{p}\left(\mathrm{y}_{2 \mathrm{n}(\mathrm{k})}, \mathrm{y}_{2 \mathrm{~m}(\mathrm{k})}\right)=\varepsilon$.

By the triangle inequality

$$
\begin{aligned}
& p\left(y_{2 n(k)}, y_{2 m(k)}\right) \leq p\left(y_{2 n(K)}, y_{2 m(k)-1}\right)+d_{2 m(k)-1} \\
& p\left(y_{2 n(K)}, y_{2 m(k)-1}\right) \leq p\left(y_{2 n(k)}, y_{2 m(k)}\right)+e_{2 m(k)-1} .
\end{aligned}
$$

So

(2.16) $\left|\mathrm{p}\left(\mathrm{y}_{2 \mathrm{n}(\mathrm{k})}, \mathrm{y}_{2 \mathrm{~m}(\mathrm{k})}\right)-\mathrm{p}\left(\mathrm{y}_{2 \mathrm{n}(\mathrm{k})}, \mathrm{y}_{2 \mathrm{~m}(\mathrm{k})-1}\right)\right| \leq \max \left\{\mathrm{d}_{2 \mathrm{~m}(\mathrm{k})-1}, \mathrm{e}_{2 \mathrm{~m}(\mathrm{k})-1}\right\}$.

Similarly by triangle inequality

$$
\left|p\left(y_{2 n(k)+1}, y_{2 m(k)-1}\right)-p\left(y_{2 n(k)}, y_{2 m(k)}\right)\right| \leq \max \left\{e_{2 n(k)}+e_{2 m(k)-1}, d_{2 n(k)}+d_{2 m(k)-1}\right\} .
$$

From (2.16) and (2.17) as $\mathrm{k} \rightarrow \infty,\left\{\mathrm{p}\left(\mathrm{y}_{2 \mathrm{n}(\mathrm{K})}, \mathrm{y}_{2 \mathrm{~m}(\mathrm{k})-1)}\right\}\right.$ and $\mathrm{p}\left(\mathrm{y}_{2 \mathrm{n}(\mathrm{k})+1}, \mathrm{y}_{2 \mathrm{~m}(\mathrm{k})-1)}\right\}$ converge to $\varepsilon$. Similarly if $\mathrm{p}\left(\mathrm{y}_{2 \mathrm{~m}(\mathrm{k})}, \mathrm{y}_{2 \mathrm{n}(\mathrm{k})}\right)>\varepsilon$,

$$
\begin{aligned}
\lim _{n \rightarrow \infty} p\left(y_{2 m(k)}, y_{2 n(k)}\right) & =\lim _{n \rightarrow \infty} p\left(y_{2 m(k)-1}, y_{2 n(k)+1}\right) \\
& =\lim _{n \rightarrow \infty} p\left(y_{2 m(k)-1}, y_{2 n(k)}\right)=\varepsilon \text { as } k \rightarrow \infty .
\end{aligned}
$$

By (2.3),

$$
\begin{aligned}
& \varepsilon<\mathrm{p}\left(\mathrm{y}_{2 \mathrm{n}(\mathrm{k})}, \mathrm{y}_{2 \mathrm{~m}(\mathrm{k})}\right) \\
& \leq \mathrm{p}\left(\mathrm{y}_{2 \mathrm{n}(\mathrm{k})}, \mathrm{y}_{2 \mathrm{n}(\mathrm{k})+1}\right)+\mathrm{p}\left(\mathrm{y}_{2 \mathrm{n}(\mathrm{k})+1}, \mathrm{y}_{2 \mathrm{~m}(\mathrm{k})}\right) \\
& \leq \mathrm{d}_{2 \mathrm{n}(\mathrm{k})}+\max \left\{\mathrm{p}\left(\mathrm{y}_{2 \mathrm{n}(\mathrm{k})+1}, \mathrm{y}_{2 \mathrm{~m}(\mathrm{k})}\right), \mathrm{p}\left(\mathrm{y}_{2 \mathrm{n}(\mathrm{k})}, \mathrm{y}_{2 \mathrm{n}(\mathrm{k})+1}\right)\right\} \\
& =\mathrm{d}_{2 \mathrm{n}(\mathrm{k})}+\max \left\{\mathrm{p}\left(\mathrm{Mx}_{2 \mathrm{n}(\mathrm{k})+1}, \mathrm{Lx}_{2 \mathrm{~m}(\mathrm{k})}\right), \mathrm{p}\left(\mathrm{Lx}_{2 \mathrm{~m}(\mathrm{k})}, \mathrm{Mx}_{2 \mathrm{n}(\mathrm{k})+1}\right)\right\} \\
& \leq \mathrm{d}_{2 \mathrm{n}(\mathrm{k})}+\left[\phi \left\{\mathrm{p}\left(\mathrm{y}_{2 \mathrm{~m}(\mathrm{k})-1}, \mathrm{y}_{2 \mathrm{~m}(\mathrm{k})}\right) \mathrm{p}\left(\mathrm{y}_{2 \mathrm{n}(\mathrm{k})}, \mathrm{y}_{2 \mathrm{n}(\mathrm{k})+1}\right),\right.\right. \\
& \quad \mathrm{p}\left(\mathrm{y}_{2 \mathrm{~m}(\mathrm{k})-1}, \mathrm{y}_{2 \mathrm{n}(\mathrm{k})+1}\right) \mathrm{p}\left(\mathrm{y}_{2 \mathrm{n}(\mathrm{k})}, \mathrm{y}_{2 \mathrm{~m}(\mathrm{k})}\right), \mathrm{p}\left(\mathrm{y}_{2 \mathrm{~m}(\mathrm{k})-1}, \mathrm{y}_{2 \mathrm{~m}(\mathrm{k})}\right) \mathrm{p}\left(\mathrm{y}_{2 \mathrm{~m}(\mathrm{k})-1}, \mathrm{y}_{2 \mathrm{n}(\mathrm{k})+1}\right), \\
& \quad \mathrm{p}\left(\mathrm{y}_{2 \mathrm{n}(\mathrm{k})}, \mathrm{y}_{2 \mathrm{~m}(\mathrm{k})}\right) \mathrm{p}\left(\mathrm{y}_{2 \mathrm{n}(\mathrm{k})}, \mathrm{y}_{2 \mathrm{n}(\mathrm{k})+1}\right), \mathrm{p}\left(\mathrm{y}_{2 \mathrm{n}(\mathrm{k})}, \mathrm{y}_{2 \mathrm{~m}(\mathrm{k})}\right) \mathrm{p}\left(\mathrm{y}_{2 \mathrm{~m}(\mathrm{k})-1}, \mathrm{y}_{2 \mathrm{~m}(\mathrm{k})}\right), \\
& \left.\quad \mathrm{p}\left(\mathrm{y}_{2 \mathrm{n}(\mathrm{k})}, \mathrm{y}_{2 \mathrm{n}(\mathrm{k})+1}\right) \mathrm{p}\left(\mathrm{y}_{2 \mathrm{~m}(\mathrm{k})-1}, \mathrm{y}_{2 \mathrm{n}(\mathrm{k})+1)}\right\}\right]^{1 / 2} .
\end{aligned}
$$

Since $\phi$ is upper semi-continuous, as $\mathrm{k} \rightarrow \infty$ we get that $\varepsilon \leq\left\{\phi\left(0, \varepsilon^{2}, 0,0,0,0\right)\right\}^{1 / 2}<\varepsilon$, which is a contradiction. Therefore $\left\{y_{n}\right\}$ is P-Cauchy sequence in $X$. Since $X$ is complete there exists a point $z$ in $X$ such that $\lim _{\mathrm{n} \rightarrow \infty} \mathrm{y}_{\mathrm{n}}=\mathrm{z}$.

$$
\lim _{\mathrm{n} \rightarrow \infty} \mathrm{Jx}_{2 \mathrm{n}}=\lim _{\mathrm{n} \rightarrow \infty} \mathrm{Mx}_{2 \mathrm{n}-1}=\mathrm{z}
$$

and

$$
\lim _{\mathrm{n} \rightarrow \infty} \mathrm{Kx}_{2 \mathrm{n}+1}=\lim _{\mathrm{n} \rightarrow \infty} \mathrm{Lx}_{2 \mathrm{n}-2}=\mathrm{z} \text {. }
$$

Since $\mathrm{L}(\mathrm{X}) \subseteq \mathrm{K}(\mathrm{X})$, there exist a point $\mathrm{u} \in \mathrm{X}$ such that $\mathrm{z}=\mathrm{Ku}$. Then using (2.3),

$$
\begin{aligned}
& \max \left\{\mathrm{p}^{2}\left(\mathrm{Lx}_{2 \mathrm{n}}, \mathrm{Mu}\right), \mathrm{p}^{2}\left(\mathrm{Mu}, \mathrm{Lx}_{2 \mathrm{n}}\right)\right\} \\
& \leq \phi\left\{\mathrm{p}\left(\mathrm{Jx}_{2 \mathrm{n}}, \mathrm{Lx}_{2 \mathrm{n}}\right) \mathrm{p}(\mathrm{Ku}, \mathrm{Mu}), \mathrm{p}\left(\mathrm{Jx}_{2 \mathrm{n}}, \mathrm{Mu}\right) \mathrm{p}\left(\mathrm{Ku}, \mathrm{Lx}_{2 \mathrm{n}}\right),\right. \\
& \mathrm{p}\left(\mathrm{Jx}_{2 \mathrm{n}}, \mathrm{Lx}_{2 \mathrm{n}}\right) \mathrm{p}\left(\mathrm{Jx}_{2 \mathrm{n}}, \mathrm{Mu}\right), \mathrm{p}\left(\mathrm{Ku}, \mathrm{Lx}_{2 \mathrm{n}}\right) \mathrm{p}(\mathrm{Ku}, \mathrm{Mu}), \\
& \left.\mathrm{p}\left(\mathrm{Ku}, \mathrm{Lx}_{2 \mathrm{n}}\right) \mathrm{p}\left(\mathrm{Jx}_{2 \mathrm{n}}, \mathrm{Lx}_{2 \mathrm{n}}\right), \mathrm{p}(\mathrm{Ku}, \mathrm{Mu}) \mathrm{p}\left(\mathrm{Jx}_{2 \mathrm{n}}, \mathrm{Mu}\right)\right\} \text {. }
\end{aligned}
$$

Taking limit as $n \rightarrow \infty$, 
$\max \left\{\mathrm{p}^{2}(\mathrm{z}, \mathrm{Mu}), \mathrm{p}^{2}(\mathrm{Mu}, \mathrm{z})\right\}$

$$
\begin{aligned}
& \leq \phi\{\mathrm{p}(\mathrm{z}, \mathrm{z}) \mathrm{p}(\mathrm{z}, \mathrm{Mu}), \mathrm{p}(\mathrm{z}, \mathrm{Mu}) \mathrm{p}(\mathrm{z}, \mathrm{z}), \mathrm{p}(\mathrm{z}, \mathrm{z}) \mathrm{p}(\mathrm{z}, \mathrm{Mu}), \\
& \quad \mathrm{p}(\mathrm{z}, \mathrm{z}) \mathrm{p}(\mathrm{z}, \mathrm{Mu}), \mathrm{p}(\mathrm{z}, \mathrm{z}) \mathrm{p}(\mathrm{z}, \mathrm{z}), \mathrm{p}(\mathrm{z}, \mathrm{Mu}) \mathrm{p}(\mathrm{z}, \mathrm{Mu})\} \\
& \leq \phi\{0,0,0,0,0, \mathrm{p}(\mathrm{z}, \mathrm{Mu}) \mathrm{p}(\mathrm{z}, \mathrm{Mu})\}, \\
& <\mathrm{p}(\mathrm{z}, \mathrm{Mu}) \mathrm{p}(\mathrm{z}, \mathrm{Mu})
\end{aligned}
$$

a contradiction. Thus $\mathrm{Mu}=\mathrm{z}$. Therefore $\mathrm{Mu}=\mathrm{z}=\mathrm{Ku}$.

Similarly, since $\mathrm{M}(\mathrm{X}) \subseteq \mathrm{J}(\mathrm{X})$, there exist a point $\mathrm{v} \in \mathrm{X}$, such that $\mathrm{z}=\mathrm{Jv}$.

Then using (2.3),

$$
\begin{aligned}
& \max \left\{\mathrm{p}^{2}\left(\mathrm{Lv}, \mathrm{Mx}_{2 \mathrm{n}+1}\right), \mathrm{p}^{2}\left(\mathrm{Mx}_{2 \mathrm{n}+1}, \mathrm{Lv}\right)\right\} \\
& \leq \phi\left\{\mathrm{p}(\mathrm{Jv}, \mathrm{Lv}) \mathrm{p}\left(\mathrm{Kx}_{2 \mathrm{n}+1}, \mathrm{Mx}_{2 \mathrm{n}+1}\right), \mathrm{p}\left(\mathrm{Jv}, \mathrm{Mx}_{2 \mathrm{n}+1}\right) \mathrm{p}\left(\mathrm{Kx}_{2 \mathrm{n}+1}, \mathrm{Lv}\right),\right. \\
& \mathrm{p}(\mathrm{Jv}, \mathrm{Lv}) \mathrm{p}\left(\mathrm{Jv}, \mathrm{Mx}_{2 \mathrm{n}+1}\right), \mathrm{p}\left(\mathrm{Kx}_{2 \mathrm{n}+1}, \mathrm{Lv}\right) \mathrm{p}\left(\mathrm{Kx}_{2 \mathrm{n}+1}, \mathrm{Mx}_{2 \mathrm{n}+1}\right) \text {, } \\
& \left.\mathrm{p}\left(\mathrm{Kx}_{2 \mathrm{n}+1}, \mathrm{Lv}\right) \mathrm{p}(\mathrm{Jv}, \mathrm{Lv}), \mathrm{p}\left(\mathrm{Kx}_{2 \mathrm{n}+1}, \mathrm{Mx}_{2 \mathrm{n}+1}\right) \mathrm{p}\left(\mathrm{Jv}_{\mathrm{v}}, \mathrm{Mx}_{2 \mathrm{n}+1}\right)\right\} \text {. }
\end{aligned}
$$

Taking limit as $\mathrm{n} \rightarrow \infty$,

$$
\begin{aligned}
& \max \left\{\mathrm{p}^{2}(\mathrm{Lv}, \mathrm{z}), \mathrm{p}^{2}(\mathrm{z}, \mathrm{Lv})\right\} \\
& \leq \phi\{\mathrm{p}(\mathrm{z}, \mathrm{Lv}) \mathrm{p}(\mathrm{z}, \mathrm{z}), \mathrm{p}(\mathrm{z}, \mathrm{z}) \mathrm{p}(\mathrm{z}, \mathrm{Lv}), \mathrm{p}(\mathrm{z}, \mathrm{Lv}) \mathrm{p}(\mathrm{z}, \mathrm{z}), \mathrm{p}(\mathrm{z}, \mathrm{Lv}) \mathrm{p}(\mathrm{z}, \mathrm{z}) \\
& \quad \mathrm{p}(\mathrm{z}, \mathrm{Lv}) \mathrm{p}(\mathrm{z}, \mathrm{Lv}), \mathrm{p}(\mathrm{z}, \mathrm{z}) \mathrm{p}(\mathrm{z}, \mathrm{z})\} \\
& \leq \phi\{0,0,0,0, \mathrm{p}(\mathrm{z}, \mathrm{Lv}) \mathrm{p}(\mathrm{z}, \mathrm{Lv}), 0\} \\
& \quad<\mathrm{p}(\mathrm{z}, \mathrm{Lv}) \mathrm{p}(\mathrm{z}, \mathrm{Lv})
\end{aligned}
$$

a contradiction. Thus $\mathrm{z}=\mathrm{Lv}$. Therefore $\mathrm{z}=\mathrm{Lv}=\mathrm{Jv}$.

Hence, $\mathrm{z}=\mathrm{Ku}=\mathrm{Mu}=\mathrm{Jv}=\mathrm{Lv}$.

Since the pair of mappings $\mathrm{K}$ and $\mathrm{M}$ are occasionally weakly compatible, then $\mathrm{KMu}=\mathrm{MKu}$.

i.e. $\mathrm{Kz}=\mathrm{Mz}$. Now we show that $\mathrm{z}$ is a fixed point of $\mathrm{M}$.

If $\mathrm{Mz} \neq \mathrm{z}$, then by $(2.3)$

$$
\begin{aligned}
& \max \left\{\mathrm{p}^{2}\left(\mathrm{Lx}_{2 \mathrm{n}}, \mathrm{Mz}\right), \mathrm{p}^{2}\left(\mathrm{Mz}, \mathrm{Lx}_{2 \mathrm{n}}\right)\right\} \\
& \leq \phi\left\{\mathrm{p}\left(\mathrm{Jx}_{2 \mathrm{n}}, \mathrm{Lx}_{2 \mathrm{n}}\right) \mathrm{p}(\mathrm{Kz}, \mathrm{Mz}), \mathrm{p}\left(\mathrm{Jx}_{2 \mathrm{n}}, \mathrm{Mz}\right) \mathrm{p}\left(\mathrm{Kz}, \mathrm{Lx}_{2 \mathrm{n}}\right),\right. \\
& \mathrm{p}\left(\mathrm{Jx}_{2 \mathrm{n}}, \mathrm{Lx}_{2 \mathrm{n}}\right) \mathrm{p}\left(\mathrm{Jx}_{2 \mathrm{n}}, \mathrm{Mz}\right), \mathrm{p}\left(\mathrm{Kz}, \mathrm{Lx}_{2 \mathrm{n}}\right) \mathrm{p}(\mathrm{Kz}, \mathrm{Mz}) \text {, } \\
& \left.\mathrm{p}\left(\mathrm{Kz}, \mathrm{Lx}_{2 \mathrm{n}}\right) \mathrm{p}\left(\mathrm{Jx}_{2 \mathrm{n}}, \mathrm{Lx}_{2 \mathrm{n}}\right), \mathrm{p}(\mathrm{Kz}, \mathrm{Mz}) \mathrm{p}\left(\mathrm{Jx}_{2 \mathrm{n}}, \mathrm{Mz}\right)\right\} \text {. }
\end{aligned}
$$

Taking limit as $\mathrm{n} \rightarrow \infty$,

$$
\begin{aligned}
& \max \left\{\mathrm{p}^{2}(\mathrm{z}, \mathrm{Mz}), \mathrm{p}^{2}(\mathrm{Mz}, \mathrm{z})\right\} \\
& \leq \phi\{\mathrm{p}(\mathrm{z}, \mathrm{z}) \mathrm{p}(\mathrm{Kz}, \mathrm{Mz}), \mathrm{p}(\mathrm{z}, \mathrm{Mz}) \mathrm{p}(\mathrm{Kz}, \mathrm{z}), \mathrm{p}(\mathrm{z}, \mathrm{z}) \mathrm{p}(\mathrm{z}, \mathrm{Mz}), \mathrm{p}(\mathrm{Kz}, \mathrm{z}) \mathrm{p}(\mathrm{Kz}, \mathrm{Mz}), \\
&\mathrm{p}(\mathrm{Kz}, \mathrm{z}) \mathrm{p}(\mathrm{z}, \mathrm{z}), \mathrm{p}(\mathrm{Kz}, \mathrm{Mz}) \mathrm{p}(\mathrm{z}, \mathrm{Mz})\} \\
& \leq \phi\{0,0,0,0,0, \mathrm{p}(\mathrm{z}, \mathrm{Mz}) \mathrm{p}(\mathrm{z}, \mathrm{Mz})\} \\
&< \mathrm{p}(\mathrm{z}, \mathrm{Mz}) \mathrm{p}(\mathrm{z}, \mathrm{Mz})
\end{aligned}
$$

a contradiction. Thus $\mathrm{Mz}=\mathrm{z}$. Therefore $\mathrm{Mz}=\mathrm{z}=\mathrm{Kz}$.

Similarly we prove that $\mathrm{Lz}=\mathrm{z}=\mathrm{Jz}$.

Hence $\mathrm{Jz}=\mathrm{Kz}=\mathrm{Lz}=\mathrm{Mz}=\mathrm{z}$; thus $\mathrm{z}$ is a common fixed point of $\mathrm{J}, \mathrm{K}, \mathrm{L}$ and $\mathrm{M}$. Uniqueness follows trivially. Therefore $\mathrm{z}$ is a unique common fixed point of $\mathrm{J}, \mathrm{K}, \mathrm{L}$ and $\mathrm{M}$. 


\section{CONClusion}

Concept of occasionally weakly compatible mappings introduced by Al-Thagafi et. al. [1] is more general than the concept of weakly compatible mappings. It is however, worth to mention here that if we take weak compatibility aspect instead of the occasionally weak compatibility, we can get the results of Rao et. al. [8,9]. Aforesaid results illustrate our assertion in the Quasi-gauge space theory. This is, however also true for the study of fixed points in metric space.

\section{REFERENCES}

[1] Al-Thagafi, M. A. and Shahzad, N., Generalized I-nonexpansive selfmaps and invariant approximations, Acta Mathematica Sinica, English Series, Vol. 24 (2008), 867-876.

[2] Antony, J., Studies in fixed points and Quasi-Gauges, Ph.D. Thesis, I.I.T., Madras(1991).

[3] Antony, Jessy and Subrahmanyam, P.V., Quasi-Gauges and Fixed Points, Univ. u Novom Sadu Zb. Rad. Prirod.-Mat. Fak. Ser. Mat. 24 (1), (1994), 31-42.

[4] Jain, A., Gupta, V.K. and Bamniya, D., Common fixed point theorem for finite number of weakly compatible mappings in Quasi-Gauge space, International Journal of Research and Reviews in Applied Sciences, 19(3), (2014), 212-218.

[5] Fisher, B., Theorems on common fixed points, Fund. Math. 113 (1981), 37-43.

[6] Matkowski, J.,Fixed point theorems for mappings with a contractive iterate at a point, Proc. Amer. Math. Soc. 62 (1977), No.2, 344-348.

[7] Pathak, H. K., Chang, S. S. and Cho, Y. J., Fixed point theorems for compatible mappings of type (P), Indian J. Math. 36 (1994), No. 2, 151-166.

[8] Rao, I. H. N. and Murty, A. S. R., Common fixed point of weakly compatible mappings in Quasi-gauge spaces, J. Indian Acad. Math. 21(1999), No. 1, 73-87.

[9] Rao, I. H. N., Rao, G.V. and Rajesh, S., Common fixed point theorems in Quasi-Gauge spaces for six self maps, International Journal of Mathematics Trends and Technology, Vol. 5 (2014), 156-167.

[10] Reilly, I.L., Quasi-gauge spaces. J. Lond. Math. Soc. 6 (1973), 481-487.

\section{AUTHORS' BIOGRAPHY}

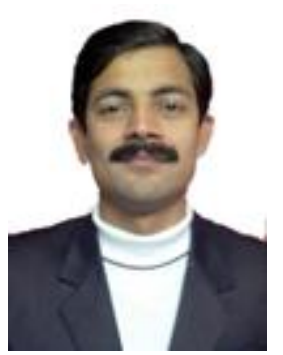

Dr. Arihant Jain, did his Post Graduation in Mathematics in the year 2000 from School of Studies in Mathematics, Vikram University, Ujjain. He has been awarded doctorate degree in Mathematics in the year 2007 from School of Studies in Mathematics, Vikram University, Ujjain on Fixed Point Theory. He has published 105 research papers in national and international journals of repute and attended and presented papers in various national and international conferences, workshops etc.. He has a post graduate teaching experience of 8 years and graduate teaching experience of 8 years. Three students have got $\mathrm{Ph} . \mathrm{D}$. under his guidance. He has also written a book on 'Right to Education'. Presently, he is working on the post of Academic Dean, Professor and Head, Department of Applied Mathematics, Shri Guru Sandipani Institute of Technology and Science, Ujjain and Principal, Shri Guru Sandipani Girls' Institute of Professional Studies, Ujjain. He is actively engaged with his researchers.

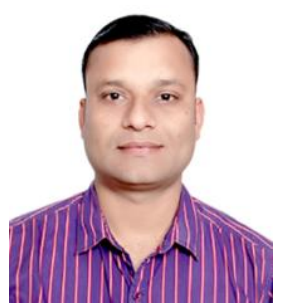

Mr. Basant Chaudhary did his Post Graduation in Mathematics in the year 2007 from D.A.V.V. Indore and M.Phil. (Mathematics) in 2008 from School of Studies in Mathematics, Vikram University, Ujjain. He has a teaching experience of 9 years. He has published a number of research papers in national as well as international journals. $\mathrm{He}$ also took active participation in national and international conferences and workshops. Presently he is engaged with his Doctoral work. 